\title{
Biologia populacional e reprodutiva de Callinectes danae Smith (Crustacea, Portunidae), no Balneário Shangri-lá, Pontal do Paraná, Paraná, Brasil 1
}

\author{
Cassiana Baptista-Metri ${ }^{2}$, Marcelo A. A. Pinheiro ${ }^{3}$, Arno Blankensteyn ${ }^{4} \&$ Carlos A. Borzone ${ }^{5}$ \\ ${ }^{1}$ Contribuição número 1493 do Departamento de Zoologia, Universidade Federal do Paraná. \\ 2 Departamento de Zoologia, Universidade Federal do Paraná. Caixa Postal 19020, 81531-980 Curitiba, Paraná, Brasil. \\ E-mail: cassian@ufpr.br \\ ${ }^{3}$ Grupo de Pesquisa em Biologia de Crustáceos, Universidade Estadual Paulista. Campus do Litoral Paulista, Praça Infante \\ Dom Henrique, 11330-900 São Vicente, São Paulo, Brasil. \\ ${ }^{4}$ Departamento de Ecologia e Zoologia, Centro de Ciências Biológicas, Universidade Federal de Santa Catarina. Caixa Postal \\ 47, 688010-970 Florianópolis, Santa Catarina, Brasil. \\ ${ }_{5}^{5}$ Centro de Estudos do Mar, Universidade Federal do Paraná. Avenida Beira Mar, 83255-000 Pontal do Paraná, Paraná, Brasil.
}

\begin{abstract}
Populational and reproductive biology of $C$. danae Smith, (Crustacea, Portunidae) in Shangrilá Beach, Pontal do Paraná, Paraná, Brazil. From April 2000 to April 2001 all individuals of C. danae Smith, 1869 included on one otter-trawl by-catch was fortnightly sampled at Shangri-lá's fish market ( $25^{\circ} 37^{\prime} 30^{\prime \prime} \mathrm{S}, 48^{\circ} 25^{\prime} \mathrm{O} 8^{\prime \prime} \mathrm{W}$ ) in order to study their population structure and reproductive pattern. Each specimen was sexed and measured $(\mathrm{CW}=$ carapace width, excluding lateral spines; $\mathrm{WW}=$ wet weight $)$. The gonadal maturation stage was determined macroscopically based on its color and size. The egg mass of the females was separate for fecundity analysis. Prevalence of adult females (including ovigerous ones) was observed comparing to other categories. Sexual proportion was 0.9:1 (males:females). Size at first sexual maturation was estimated at $60.5 \mathrm{~mm}$ for males, and $52.7 \mathrm{~mm}$ for females. WW/WC relationship was isometric for males and females. The carapace width of ovigerous females was between 43.2 to $79.5 \mathrm{~mm}(67 \pm 8 \mathrm{~mm})$, and fecundity varied from 25,127 to 246,676 eggs, with an average of 127,177 eggs per female and mean egg diameter was $0.22 \mathrm{~mm}$. The highest presence of ovigerous females occurred during winter and summer.

KEY-WORDS. Fecundity, population biology, seabob by-catch, Sex-ratio, swimming crab.
\end{abstract}

RESUMO. Entre abril/ 2000 a abril/ 2001 todos os indivíduos de $C$. danae Smith, 1869 procedentes de um mesmo arrasto foram coletados em visitas quinzenais às bancas dos pescadores do Balneário Shangri-lá $\left(25^{\circ} 37^{\prime} 30^{\prime \prime} S\right.$, $48^{\circ} 25^{\prime} 08^{\prime \prime} \mathrm{W}$ ) visando investigar sua estrutura populacional e parâmetros reprodutivos. De cada indivíduo foi determinado o sexo, a largura cefalotorácica (LC) e o peso úmido (P). O estágio de maturação gonadal foi determinado macroscopicamente pelo aspecto e coloração das gônadas. A massa de ovos das fêmeas foi separada para análise da fecundidade. Foi observado um predomínio de fêmeas adultas (incluindo as ovígeras) em relação às outras categorias. A proporção sexual foi 0,9:1 (machos:fêmeas). O tamanho de primeira maturação sexual foi estimado em $60,5 \mathrm{~mm}$ (machos) e $52,7 \mathrm{~mm}$ (fêmeas). A relação LC/P foi isométrica para machos e fêmeas. A largura da carapaça das fêmeas ovígeras variou de 43,2 a 79,5 mm (67 $\pm 8 \mathrm{~mm}$ ), com fecundidade variando entre 25.127 e 246.676 ovos, com uma média de 127.177 ovos por fêmea. O diâmetro médio dos ovos foi de $0,22 \mathrm{~mm}$. A maior incidência de fêmeas ovígeras ocorreu durante o inverno e o verão.

PALAVRAS CHAVE. Fecundidade, biologia populacional, fauna acompanhante, razão sexual, siris.

Callinectes danae Smith, 1869 distribui-se da Flórida (EUA) até o Rio Grande do Sul (Brasil), sendo registrada da região entremarés até $75 \mathrm{~m}$ de profundidade. Apresenta grande tolerância à salinidade, podendo ocupar áreas estuarinas, particularmente aquelas com sedimento lodoso (Melo 1999).
No Brasil, o conhecimento biológico sobre essa espécie tem respaldo nas contribuições de PITA et al. (1985a), que estudaram a distribuição e tamanho de primeira maturação, além dos estudos desenvolvidos por Branco \& Thives (1991), Branco et al. (1992a, b), Branco \& Masunari $(1992,2000)$, Branco \& Avilar

Revista Brasileira de Zoologia 22 (2): 446-453, junho 2005 
(1992), Branco (1996a, b) e Branco \& Masunari (2000), que analisaram aspectos da sua biologia no Estado de Santa Catarina. Na região de Ubatuba (São Paulo), o ciclo reprodutivo de C. danae foi estudado por Costa \& Negreiros-Fransozo $(1996,1998)$, com base na fecundidade, desenvolvimento gonadal e ciclo de muda.

No litoral do Paraná, C. danae e Callinectes sapidus Rathbun, 1896 são recursos pesqueiros explorados intensamente dentro da Baía de Paranaguá, possibilitando a complementação alimentar e econômica das comunidades litorâneas (IвAмA 1995). No Balneário Shangri-lá, C. danae é o segundo portunídeo mais abundante no rejeito do camarão Xiphopenaeus kroyeri Heller, 1862, sendo descartado juntamente com Callinectes ornatus Ordway, 1863 e diversas espécies de peixes jovens, caracterizando o expressivo impacto dessa modalidade pesqueira (AlvERson et al. 1994).

O presente estudo traz informações sobre a biologia populacional de $C$. danae (abundância mensal, proporção sexual e relação peso/tamanho), bem como aspectos de sua reprodução (maturidade, fecundidade e época reprodutiva), visando contribuir com futuros programas locais de gerenciamento e manejo deste importante componente do rejeito da pesca camaroeira paranaense.

\section{MATERIAL E MÉTODOS}

Os siris foram coletados quinzenalmente de abril/2000 a abril/2001, junto às bancas dos pescadores do Balneário Shangrilá $\left(25^{\circ} 37^{\prime} 30^{\prime \prime} \mathrm{S}, 48^{\circ} 25^{\prime} 08^{\prime \prime} \mathrm{W}\right)$, provenientes de um único arrasto comercial com rede de arrasto de portas, malha de $2 \mathrm{~cm}$ entrenós opostos e uma hora de duração. Os exemplares foram identificados e sexados segundo Williams (1974) e Melo (1996), mensurados com paquímetro (LC = largura cefalotorácica entre a base dos espinhos laterais) e pesados em balança com precisão de $1 \mathrm{~g}$ ( $\mathrm{P}$ = peso úmido). A fase de desenvolvimento (jovem e adulta) de cada espécime foi obtida pela morfologia do abdome e sua aderência aos esternitos torácicos (selado nos jovens e livre nos adultos). Após dissecação, cada indivíduo foi categorizado quanto ao estágio de desenvolvimento gonadal, conforme descrição realizada por PinheIro \& Fransozo (1998).

Somente as fêmeas com ovos em estágio inicial de desenvolvimento, determinadas de acordo com Costa \& NegreIRos-Fransozo (1996), foram utilizadas na estimativa da fecundidade. Os ovos foram removidos dos pleópodos (vide CHоy 1985), com retirada de três subamostras de ovos $(n=50)$ de diferentes regiões da massa ovígera (externa, mediana e interna), para a determinação de seu diâmetro com microscópio óptico (1000x) acoplado a um sistema de análise de imagens (Sigma Scan Pro, Versão 2.0). As massas ovígeras foram desidratadas em estufa $\left(80^{\circ} \mathrm{C}\right.$ por 36 horas), sendo reservadas três subamostras de aproximadamente mil ovos, que foram pesadas em balança analítica $(0,0001 \mathrm{~g})$ e a fecundidade individual estabelecida por regra de três simples.

A proporção sexual (machos:fêmeas) foi calculada mensalmente e totalizada para o período de coleta, sendo utilizado o teste de Qui-quadrado $\left(\chi^{2}\right)$ para testar a diferença de 1:1.
A curva de maturidade fisiológica foi determinada pelo percentual de indivíduos adultos (gônadas em maturação e maturas) de cada sexo nas classes de tamanho, sendo as freqüências de adultos (\%) a variável dependente e as classes de tamanho (LC) a independente. Os pontos empíricos receberam ajuste pela ogiva de Galton $\left(y=1-e^{-A Z}\right.$, onde $\left.Z=x^{b}\right)$ e $o$ tamanho de início de maturação sexual obtido por $\mathrm{LC}_{50 \%}=\mathrm{e}^{[\ln [-\ln 0,500]-\ln \mathrm{A}] / \mathrm{b}}$ (FonTeLES-FiLHo 1989).

A relação do peso úmido pela largura cefalotorácica (P/LC) foi estabelecida para cada sexo e submetida à análise de regressão, com verificação do ajuste dos pontos empíricos à função potência $\left(\mathrm{Y}=\mathrm{aX} \mathrm{X}^{\mathrm{b}}\right)$ e significância estabelecida pelo teste t. Os indivíduos danificados ou com organismos epibiontes foram excluídos das análises e a taxa de crescimento em peso foi avaliada pelo grau de alometria e confirmada pelo teste $t$, como isométrico $(\mathrm{b}=3)$, alométrico positivo $(\mathrm{b}>3)$ ou alométrico negativo (b < 3) (HARTNOLL 1982).

O período reprodutivo da espécie foi determinado pelo percentual mensal de fêmeas ovígeras em relação ao total de adultas no mês, durante um ano de estudos. O número de ovos (NO) foi determinado para cada fêmea ovígera e relacionado ao seu tamanho (LC), sendo os pontos da relação submetidos à análise de regressão e significância estatística avaliada pelo teste t.

\section{RESULTADOS}

Foram analisados 763 indivíduos de $C$. danae, representados por 372 machos (111 juvenis e 261 adultos) e 391 fêmeas (66 juvenis, 248 adultas não ovígeras e 77 ovígeras). Dentre os machos, os adultos estiveram presentes em todos os meses (Tab. I), enquanto os juvenis foram menos abundantes, não ocorrendo em julho e agosto/2000. As fêmeas adultas não ovígeras apresentaram freqüência elevada, principalmente em setembro, outubro e dezembro/2000 e março/2001, enquanto as ovígeras ocorreram na maioria dos meses, exceto em maio, agosto e novembro/2000, e em abril/2001. As fêmeas jovens foram pouco abundantes, com ocorrência esparsa durante o ano e maior incidência em janeiro/2001.

Os machos apresentaram largura cefalotorácica e peso úmido variando entre 26,1 a $93,2 \mathrm{~mm}(66,7 \pm 16,7 \mathrm{~mm})$ e 2 a $109 \mathrm{~g}(43,7 \pm 25,3 \mathrm{~g})$, respectivamente, sendo em média superiores aos valores obtidos para as fêmeas, que foram de 26,5 a $86 \mathrm{~mm}(61,0 \pm 14,4 \mathrm{~mm})$ e 2 a $81 \mathrm{~g}(32,0 \pm 17 \mathrm{~g})$.

As relações $\mathrm{P} / \mathrm{LC}$ obtidas para cada sexo foram significativas (machos: $\mathrm{t}=111,7 ; \mathrm{p}<0,05$; e fêmeas: $\mathrm{t}=89,1 ; \mathrm{p}<0,05$ ) (Figs 1-2), apresentando crescimento em peso isométrico para ambos os sexos (machos: $b=3,0 ; t=0,25 ; p>0,05$; e fêmeas: $\mathrm{b}=2,9 ; \mathrm{t}=0,18 ; \mathrm{p}>0,05)$.

A razão sexual da espécie (machos:fêmeas) foi de 0,9:1, não diferindo de 1:1 ( $\left.\chi^{2}=0,47 ; \mathrm{GL}=1 ; \mathrm{p}<0,05\right)$, embora os machos tenham sido registrados numa maior proporção em agosto e novembro/2000, e março e abril/2001 (Tab. I), enquanto as fêmeas predominaram em julho/2000, setembro/2000 e outubro/2000.

Dos 348 machos analisados quanto à maturação gonadal,

Revista Brasileira de Zoologia 22 (2): 446-453, junho 2005 

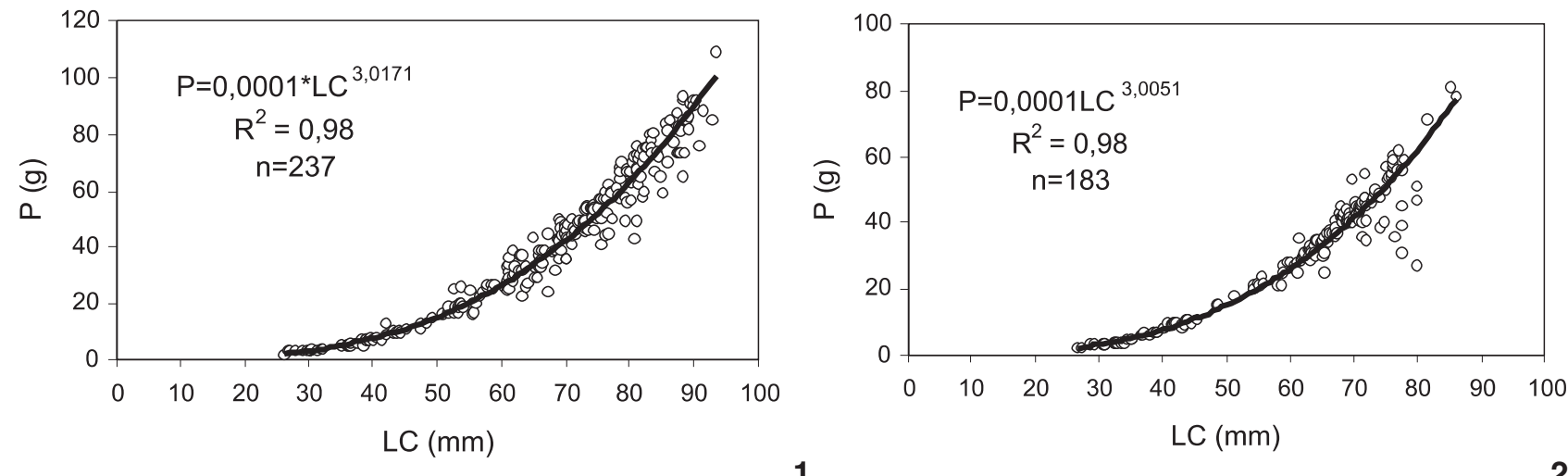

Figuras 1-2. Relação do peso úmido (P) pela largura da carapaça (LC) para os machos (1) e fêmeas (2) de Callinectes danae.

Tabela I. Número total de indivíduos machos e fêmeas em cada fase de desenvolvimento e razão sexual, durante os meses de amostragem.

\begin{tabular}{|c|c|c|c|c|c|c|c|c|c|}
\hline \multirow{3}{*}{ Mês/ano } & \multicolumn{3}{|c|}{ Machos } & \multicolumn{4}{|c|}{ Fêmeas } & \multicolumn{2}{|c|}{ Razão sexual } \\
\hline & \multirow{2}{*}{ Jovem } & \multirow{2}{*}{ Adulto } & \multirow{2}{*}{ Total } & \multirow{2}{*}{ Jovem } & \multicolumn{2}{|c|}{ Adulta } & \multirow{2}{*}{ Total } & \multirow{2}{*}{$(\mathrm{M}: \mathrm{F})$} & \multirow{2}{*}{$x^{2}$} \\
\hline & & & & & Não ovígera & Ovígera & & & \\
\hline Abril/2000 & 3 & 1 & 4 & - & 1 & 2 & 3 & $1,3: 1$ & $0,14 \mathrm{~ns}$ \\
\hline Maio & 4 & 2 & 6 & - & 10 & - & 10 & $0,6: 1$ & $1,00 \mathrm{~ns}$ \\
\hline Junho & 8 & 24 & 32 & 2 & 27 & 9 & 38 & $0,8: 1$ & $0,51 \mathrm{~ns}$ \\
\hline Julho & - & 7 & 7 & 1 & 6 & 12 & 19 & $0,4: 1$ & 5,54 * \\
\hline Agosto & - & 13 & 13 & - & 7 & - & 7 & $1,9: 1$ & $1,80 \mathrm{~ns}$ \\
\hline Setembro & 5 & 13 & 18 & 3 & 47 & 6 & 56 & $0,3: 1$ & 19,51 * \\
\hline Outubro & 2 & 23 & 25 & - & 43 & 5 & 48 & $0,5: 1$ & 7,25 * \\
\hline Novembro & 3 & 27 & 30 & - & 5 & - & 5 & $6,0: 1$ & 17,86 * \\
\hline Dezembro & 1 & 69 & 70 & - & 48 & 16 & 64 & $1,1: 1$ & $0,27 \mathrm{~ns}$ \\
\hline Janeiro/2001 & 46 & 10 & 56 & 53 & 6 & 4 & 63 & $0,9: 1$ & $0,41 \mathrm{~ns}$ \\
\hline Fevereiro & 10 & 5 & 15 & 5 & 10 & 11 & 26 & $0,6: 1$ & $2,95 \mathrm{~ns}$ \\
\hline Março & 27 & 49 & 76 & 2 & 37 & 12 & 51 & $1,5: 1$ & 4,92 * \\
\hline Abril & 2 & 18 & 20 & - & 1 & - & 1 & $20,0: 1$ & 17,19 * \\
\hline Total & 111 & 261 & 372 & 66 & 248 & 77 & 391 & $0,9: 1$ & $0,47 \mathrm{~ns}$ \\
\hline
\end{tabular}

${ }^{*} \mathrm{p}<0,05 ;$ n.s = não significativo.

102 eram imaturos $(29,1 \%), 165$ em maturação $(47,2 \%)$ e 83 maturos $(23,7 \%)$, sendo estes últimos mais abundantes em agosto e novembro/2000 e abril/2001 (Fig. 3-4). No caso das fêmeas, dos 301 exemplares analisados, 64 apresentavam gônadas imaturas (21,2\%), 66 em maturação (22\%) e 171 maturas $(56,8 \%)$. As fêmeas com gônadas maturas ocorreram em maior percentual de maio a agosto/2000, novembro/2000, com outra incidência em março/2001, quando superaram as demais categorias (Figs 3-4).

Foram utilizados 348 machos e 301 fêmeas para composição das curvas de maturidade fisiológica (Figs 5-6), que indicaram os tamanhos de início de maturidade $\left(\mathrm{LC}_{50 \%}\right)$ para cada sexo (machos: 60,5 mm; fêmeas: 52,7 mm).
Ocorreram dois picos de fêmeas ovígeras, centrados nos meses de inverno (junho e julho) e verão (dezembro a março), ocorrendo menores incidências em abril $(\mathrm{n}=3)$, setembro e outubro/2000 (Fig. 7 ).

A variação de LC nas fêmeas ovígeras foi de 43,2 a 79,4 $\mathrm{mm}(67,7 \pm 8,0 \mathrm{~mm})$, com registro para outubro e abril/2000, respectivamente. A maioria das fêmeas ovígeras apresentou tamanhos entre 65 e $75 \mathrm{~mm}$ (Fig. 8).

Das 77 ovígeras capturadas, 36 espécimes tiveram o diâmetro e o número total dos ovos determinado. As fêmeas analisadas apresentaram uma ampla variação da fecundidade (Fig. 9), com o número de ovos variando de 25.127 a 246.676 ovos/fêmea (127.177 \pm 65.305 ovos) e diâmetro entre 0,20 a $0,24 \mathrm{~mm}$ 


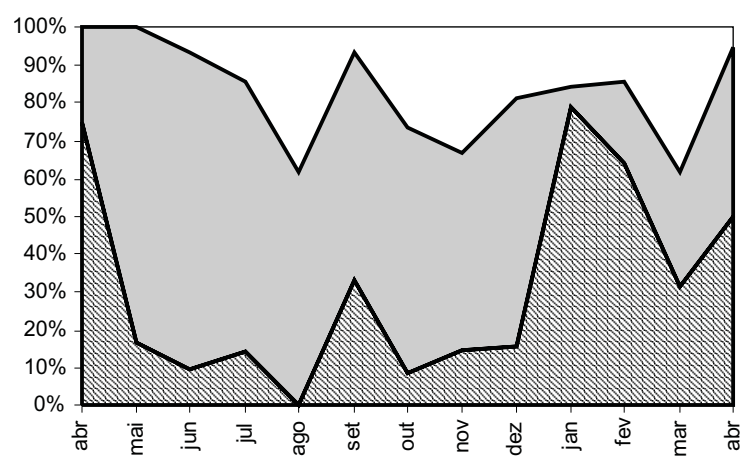

Matura 口Em Maturação $\square$ Imatura

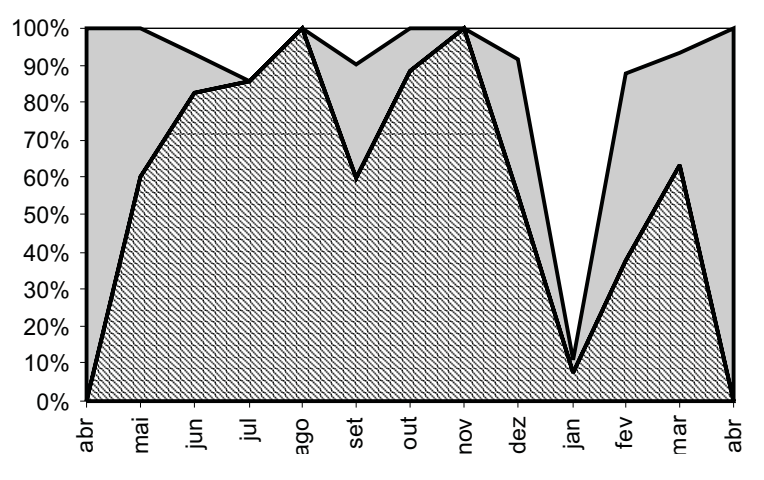

Figuras 3-4. Callinectes danae. Freqüência relativa (\%) dos estágios de maturação gonadal dos machos (3) e das fêmeas (4) de Callinectes danae, no Balneário Shangri-lá, Paraná, ao longo do período de coleta.
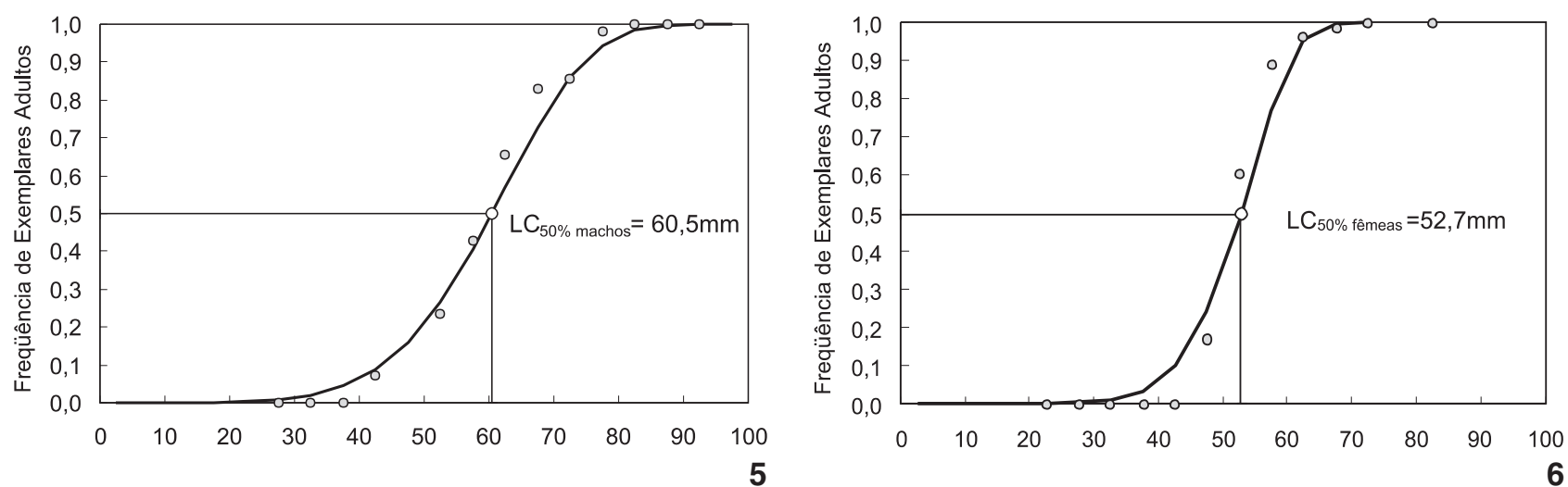

Figuras 5-6. Curvas de maturidade fisiológica dos machos (5) e fêmeas (6) de Callinectes danae, indicando o tamanho de maturação $\left(\mathrm{LC}_{50 \%}=\right.$ largura da carapaça em que metade da população é adulta).

$(0,22 \pm 0,01 \mathrm{~mm})$. A menor classe de tamanho (40 a $45 \mathrm{~mm})$ apresentou a menor fecundidade, enquanto a maior fecundidade foi registrada numa classe de tamanho intermediário (55 a 60 mm) (Fig. 10). A relação NO/LC não mostrou correlação significativa entre suas variáveis ( $\mathrm{r}=0,03 ; \mathrm{t}=1,1 ; \mathrm{p}>0,05 ; \mathrm{n}=37$ ), o mesmo ocorrendo em relação ao ajuste da função potência.

\section{DISCUSSÃO}

A pesca camaroeira com redes de arrasto tem gerado grande preocupação pelo impacto que causa às populações do necton e bentos marinho, em função de sua característica predatória e não seletiva. Após a separação dos camarões e de outros organismos de maior porte (peixes e siris), os exemplares restantes, constituídos na maioria por estágios juvenis, são descartados ainda no mar pelos pescadores. Em função disso, o estudo biológico do rejeito pesqueiro torna-se prioritário na elaboração de programas de manejo em determinada área, embora represente apenas fragmentos da população em questão, impossibilitando seu uso em estudos de biologia populacional. Este fato foi confirmado no presente trabalho, uma vez que não foi possível determinar o recrutamento, crescimento e as taxas de natalidade/mortalidade populacional de $C$. danae, em função da ausência de determinadas classes de tamanho. Os indivíduos jovens, por exemplo, são encontrados em áreas estuarinas da região (Chacur \& Negreiros-Fransozo 2001, Pita et al. 1985a), onde os pescadores de Shangri-lá não pescam.

Os dados obtidos mostram uma proporção sexual diferenciada de $C$. danae ao longo do tempo, com predomínio de fêmeas adultas (42\%) e reduzida abundância de jovens (23\%). Uma distribuição espaço/temporal diferenciada entre os sexos, com migração das fêmeas maturas para a desova, já foi confirmada para outros portunídeos, como C. sapidus (Williams 1974); C. ornatus (Pita et al. 1985a, Branco \& Lunardon-Branco 1993a, Negreiros-Fransozo \& Fransozo 1995 Negreiros-Fransozo et al. 1999); e Arenaeus cribrarius (Lamarck, 1818) (Pinheiro \& Fransozo 2002). Registros similares foram obtidos para C. danae por PitA et al. (1985b), no Complexo Estuarino-Lagunar de Santos (São Paulo), quando verificaram que as fêmeas imaturas predominam no interior da Baía, ao contrário das maturas que são registradas fora dela. Negreiros-Fransozo \& Fransozo (1995), 

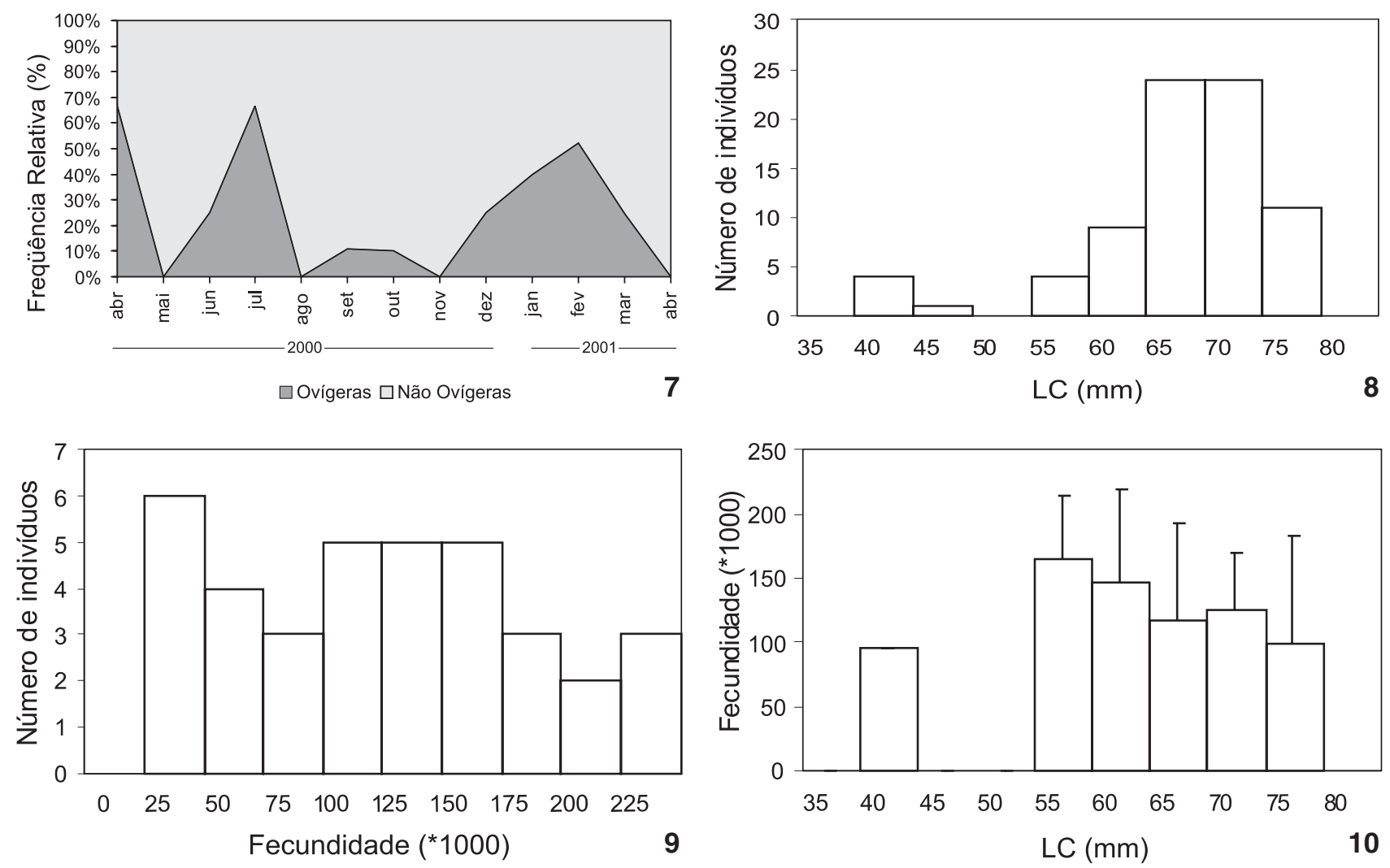

Figuras 7-10. (7) Freqüência relativa (\%) de fêmeas adultas ovígeras e não ovígeras (em abril/2000 apenas três fêmeas foram coletadas); (8-10) distribuição do número de fêmeas ovígeras em classes de: (8) largura da carapaça (LC), (9) número de ovos e (10) largura da carapaça (média e desvio padrão).

Chacur et al. (2000) e Chacur \& Negreiros-Fransozo (2001) confirmaram tais registros para $C$. danae na Baía de Ubatuba, o mesmo tendo sido reportado por BRANCo \& MASUNARI (2000) para a Lagoa da Conceição (Santa Catarina). A distribuição espaço/ temporal diferenciada entre os sexos, aliada a expressiva concentração de fêmeas ovígeras de C. danae em Shangri-lá (20\% do total de fêmeas capturadas), indica que as regiões de extração camaroeira coincidem com as áreas de desova dessa espécie. Tal fato foi similar ao relatado para as fêmeas ovígeras de outros portunídeos já estudados, que migram para áreas de maior profundidade e salinidade (CHAZARO-OlverA et al. 2000) ou visando proteção à prole, como a desembocadura de baías, lagoas ou estuários (Pita et al. 1985a, PinHeiro et al. 1997, BranCO \& Masunari 2000). Tal estratégia visa prover um ambiente mais favorável ao desenvolvimento embrionário e larval, além de favorecer a dispersão da prole.

Foram observadas fêmeas fisiologicamente maduras de C. danae ao longo de todo o ano, sendo mais freqüentes em agosto e novembro/2000 (inverno e primavera), enquanto as ovígeras apresentaram quatro incidências anuais, com picos no inverno e no verão. Tais resultados foram similares aos ob- tidos por Costa \& Negreiros-Fransozo (1998), que observaram maiores abundâncias de fêmeas maduras em julho e setembro (inverno), embora as ovígeras tenham sido registradas durante todo o ano. No entanto, na Lagoa da Conceição, BRANCO \& MASUNARI (2000) verificaram uma maior abundância nos meses de janeiro, fevereiro e junho (verão e inverno), corroborando o padrão observado no presente estudo. Segundo a classificação proposta por Pinheiro \& Fransozo (2002), os dados obtidos indicam que $C$. danae apresenta uma reprodução sazonal-contínua, ocorrendo em todos os meses do ano, mas com maior incidência em algumas estações (verão e inverno). Tal informação se coaduna com os relatos sobre esta espécie por Pita et al. (1985a), Costa \& Negreiros-Fransozo (1998) e Chacur et al. (2000), ocorrendo o mesmo com C. ornatus, Portunus spinimanus Latreille, 1819 e por $A$. cribrarius, estudados por Mantelatto \& Fransozo (1997),Santos \& Negreiros-Fransozo (1999) e Pinheiro \& Fransozo (2002), respectivamente.

A maturidade está relacionada ao alcance de um determinado tamanho individual (FonTELEs-FilHo 1989). Nos crustáceos decápodos pode variar intraespecifica e latidudinalmente em função da ação diferenciada de fatores ambientais como a 
salinidade, temperatura e luminosidade (HINEs 1989, LESTANG et al. 2003). No Balneário Shangri-lá, os tamanhos de primeira maturação para C. danae (machos: 60,5 mm; fêmeas: $52,7 \mathrm{~mm}$ ) foram pouco inferiores aos determinados por PITA et al. (1985a), em Santos (machos: $67 \mathrm{~mm}$; fêmeas: $55 \mathrm{~mm}$ ). Apesar disso, contrastam expressivamente quando confrontados com os maiores tamanhos obtidos no Estado de Santa Catarina por Branco \& Masunari (2000) (98,5 e 88,8 mm) e Branco \& Thives (1991) (94 e $84 \mathrm{~mm}$ ). O maior tamanho dos machos de $C$. danae ao atingir a maturidade representa uma adaptação reprodutiva, já que este dimorfismo confere maior proteção às fêmeas em pós-muda logo após a cópula (abraço pós-copulatório), como já relatado para outros portunídeos (Branco \& Lunardon-Branco 1993b, Mantelatto \& Fransozo 1996, Pinheiro \& Fransozo 1998, Baptista et al. 2003, Branco \& Fracasso 2004).

A comparação entre as médias de largura da carapaça (LC) e peso (P), indica que os machos apresentaram um crescimento em tamanho mais expressivo do que as fêmeas. Tal diferença sexual advêm com a muda de puberdade, quando a energia metabólica é alocada ao crescimento somático nos machos, enquanto nas fêmeas é direcionada à produção de ovos (HARTNOLL 1985, Mantelatto \& Martinelli 1999). Exemplares de C. danae procedentes de manguezal apresentaram um crescimento alométrico negativo para a relação P/LC (BRANCo \& THIVEs 1991), tornando evidente que a época, local e características ambientais podem propiciar variações no tipo de crescimento desses organismos. Tais variações também são notórias quanto ao tamanho das fêmeas ovígeras em função da similaridade verificada entre os exemplares capturados em Shangri-lá (43,2 a 79,4 mm) e aqueles provenientes de Ubatuba ( 48 a $83 \mathrm{~mm}$, segundo Costa \& NegreirosFransozo 1996), os quais, por sua vez, foram inferiores aos da Lagoa da Conceição (75 a 110 mm (Branco \& Avilar 1992).

A variação da fecundidade obtida para C. danae (25.127246.676 ovos) foi muito inferior à determinada em Ubatuba por Costa \& Negreiros-Fransozo (1996) (363.660-826.638 ovos) e em Florianópolis (Santa Catarina) por Branco \& AviLAR (1992) (111.549-1.292.190 ovos). A fecundidade obtida no presente trabalho foi independente do diâmetro dos ovos, já que esta característica é de reduzida variação intraespecífica (HARTNOLL \& Gould 1988). A média de diâmetro dos ovos (0,22 mm) não diferiu da literatura, apesar de ter sido menor que a observada por Branco \& Avilar (1992). Assim, a menor fecundidade das fêmeas de $C$. danae em Shangri-lá pode ser atribuída a diferenças nos métodos de quantificação do número de ovos, características genéticas da população ou do habitat estudado.

Na maioria dos crustáceos as fêmeas de maior porte possuem maior fecundidade (SASTRY 1983). Esta relação não foi obtida no presente trabalho $\left(\mathrm{R}^{2}=0,03 ; \mathrm{t}=1,1 ; \mathrm{n}=36\right)$, ao contrário do resultado obtido por Branco \& Avilar (1992) na Lagoa da Conceição $\left(\log \mathrm{NO}=0,75+3,58 \log \mathrm{LC} ; \mathrm{R}^{2}=0,58 ; \mathrm{n}=59\right)$ e por Costa \& Negreiros-Fransozo (1996) em Ubatuba (NO = $\left.103185,2+1,4 \mathrm{LC} ; \mathrm{R}^{2}=0,51 ; \mathrm{n}=28\right)$. Analisando o gráfico de dispersão da relação NO/LC apresentado por CosTa \& NEGREIROS-
FrANSOzo (1996) observa-se claramente dois agrupamentos com tendência linear, que, na ausência de um caráter biológico discriminante, não puderam gerar duas equações de fecundidade, o que parecia ser o caso. Além disso, os índices apresentados por estas autoras são baixos, fato similar ao reportado para outra espécie congênere (C. ornatus) por MantelatTo \& Fransozo (1997). Possivelmente este fato encontre explicação na expressiva variação do número de ovos numa mesma classe de tamanho, diferenciando fêmeas de primeira desova (primíparas) daquelas em desovas subseqüentes (multíparas), conforme já reportado para outros braquiúros, a saber: Chionoecetes bairdi Rathbun 1924 por Somerton \& Meyers (1983), PAUL (1984) e DONALDSON \& ADAMs (1989), para Paralithodes camtschaticus Tilesius 1815 por STONE et al. (1992), e para Maja squinado (Herbst 1788) por GonzÁlez-GurRiarán et al. (1998).

Apesar da maioria das espécies de braquiúros apresentar a relação NO/LC ajustada por uma única equação, a exemplo de Ucides cordatus (Linnaeus, 1763) (HATTORI \& PinHeIro 2003) e outros portunídeos: Callinectes similis Williams 1966 (CHAZARo-Olvera et al. 2000), Portunus spinimanus (Santos \& Negreiros-Fransozo 1997) e A. cribrarius (PINHEIro \& TERCEIro 2000), a ocorrência de fêmeas com ovos em estágio embrionário final e com gônadas maturas, confirma a hipótese de desova múltipla para alguns portunídeos, como mencionado para C. ornatus por MANTELATTO \& Fransozo (1997).

As informações do presente trabalho reforçam a importância de estudos sobre o impacto causado nas populações de invertebrados e peixes descartados na pesca camaroeira. Estas podem ser extrapoladas para outras regiões litorâneas, com enfoque às espécies do gênero Callinectes Stimpson, 1860 (C. danae e $C$. sapidus), freqüentemente capturadas nos setores internos da Baía de Paranaguá, já que consistem num recurso pesqueiro de subsistência e remuneração para algumas comunidades locais (Івама 1995).

\section{AGRADECIMENTOS}

Dedicamos esse trabalho a memória de Emidia de Fátima Farias, cuja inestimável ajuda possibilitou a execução das atividades de campo e realização do presente estudo. Agradecemos aos pescadores cooperados de Shangri-lá por permitirem utilizar os exemplares capturados. Ao Curso de Pós-Graduação em Zoologia da Universidade Federal do Paraná (UFPR), bem como ao Centro de Estudos do Mar (CEM), por todo apoio durante o desenvolvimento dessa pesquisa. A CAPES pela bolsa de mestrado concedida à primeira autora. A Dra. Andréa S. Freire e Msc. Nilva Brandini pelas sugestões na estimativa da fecundidade e análise na obtenção dos dados, respectivamente. À amiga Fátima S.T. Vara pelo auxílio na mensuração dos ovos.

\section{REFERÊNCIAS BIBLIOGRÁFICAS}

Alverson, D.L.; M.H. Freeberg; J.G. Pope \& S.A. Murawisk. 1994. A global assement of fisheries bycatch and discards. FAO Fisheries Technical Paper, Rome, 339: 1-233. 
Baptista, C.; M.A.A. Pinheiro; A. Blankensteyn \& C.A. Borzone. 2003. Estrutura populacional de Callinectes ornatus Ordway (Crustácea, Portunidae) no Balneário Shangri-lá, Pontal do Paraná, Paraná, Brasil. Revista Brasileira de Zoologia, Curitiba, 20 (4): 661-666.

Branco, J.O. 1996a. Ciclo e ritmo alimentar de Callinectes danae Smith, 1869 (Decapoda, Portunidae) da Lagoa da Conceição, Florianópolis, SC. Arquivos de Biologia e Tecnologia, Curitiba, 39 (4): 987-998.

BRANCO, J.O. 1996b. Variações sazonais e ontogênicas na dieta natural de Callinectes danae Smith, 1869 (Decapoda, Portunidae) da Lagoa da Conceição, Florianópolis, SC. Arquivos de Biologia e Tecnologia, Curitiba, 39 (4): 987-998.

Branco, J.O. \& A. Thives. 1991. Relação peso/largura, fator de condição e tamanho de primeira maturação de Callinectes danae Smith, 1869 (Crustacea, Portunidae) no Manguezal do Itacorubi, SC, Brasil. Arquivos de Biologia e Tecnologia, Curitiba, 34 (3/4): 415:424.

Branco J.O. \& M.G. Avilar. 1992. Fecundidade em Callinectes danae Smith, 1869 (Decapoda, Portunidae) da Lagoa da Conceição, Florianópolis, Santa Catarina, Brasil. Revista Brasileira de Zoologia, Curitiba, 9 (3/4): 167-173.

Branco, J.O. \& M. J. Lunardon-Branco. 1993a. Crescimento e tamanho de primeira maturação em Callinectes ornatus Ordway, 1863 (Decapoda, Portunidae) da região de Matinhos, Paraná, Brasil. Arquivos de Biologia e Tecnologia, Curitiba, 36 (3): 489-496.

Branco, J.O. \& M. J. Lunardon-Branco. 1993b. Crescimento e tamanho de primeira maturação em Callinectes ornatus Ordway, 1863 (Decapoda, Portunidae) da região de Matinhos, Paraná, Brasil. Arquivos de Biologia e Tecnologia, Curitiba, 36 (3): 497-503.

Branco J.O. \& S. Masunari. 1992. Crescimento de Callinectes danae Smith (Decapoda, Portunidae) da Lagoa da Conceição, Santa Catarina, Brasil. Revista Brasileira de Zoologia, Curitiba, 9 (1-2): 53-66.

Branco, J.O. \& S. Masunari. 2000. Reproductive ecology of the blue crab, Callinectes danae Smith, 1869 in the Conceição Lagoon System, Santa Catarina Isle, Brazil. Revista Brasileira de Biologia, Curitiba, 60 (1): 17-27.

Branco, J.O. \& H.A.A. Fracasso. 2004. Biologia populacional de Callinectes ornatus (Ordway) na Armação do Itapocoroy, Penha, Santa Catarina, Brasil. Revista Brasileira de Zoologia, Curitiba, 21 (1): 91-96.

Branco, J.O.; M.J. Lunardon; M. G Avila \& C.F. Miguez. 1992a. Interação entre o fator de condição e o índice gonadossomático como indicadores do período de desova em Callinectes danae Smith (Decapoda, Portunidae) da Lagoa da Conceição, Florianópolis, Santa Catarina, Brasil. Revista Brasileira de Zoologia, Curitiba, 9 (3/4): 175-180.

Branco, J.O.; M.J. Lunardon; J. Loyola e Silva \& M.G. Avila. 1992b. Observações bioecológicas sobre o "siri azul" Callinectes danae Smith, 1869 (Decapoda, Portunidae) da Baía Norte,
SC, Brasil. Arquivos de Biologia e Tecnologia, Curitiba, 35 (3): 557-546.

Chacur, M.M. \& M.L. Negreiros-Fransozo. 2001. Spatial and seasonal distributions of Callinectes danae (Decapoda, Portunidae) in Ubatuba Bay, São Paulo, Brazil. Journal of Crustacean Biology, Lawrence, 21 (2): 414-425.

Chacur, M.M.; C.B. Mansur \& M.L. Negreiros-Fransozo. 2000. Distributional patterns, seasonal abundance and moult cycle of Callinectes danae Smith, 1869 in the Ubatuba region, Brazil. Nauplius, Botucatu, 8 (2): 215-226.

Chazaro-olvera, S.; A. Rocha-ramirez \& R. Roman-Contreras. 2000. Observations on feeding, maturity, and fecundity of Callinectes similis Williams, 1966 on the central continental shelf off Vera Cruz, Gulf of Mexico. Crustaceana, Leiden, 73 (3): 323-332.

Choy, S.C. 1985. A rapid method for removing and couting eggs from fresh and preserved decapod cruastaceans. Aquaculture, Amsterdam, 48: 369-372.

Costa, T.M. \& M.L. Negreiros-Fransozo. 1996. Fecundidade de Callinectes danae Smith, 1869 (Crustacea, Decapoda, Portunidae) na região de Ubatuba (SP), Brasil. Brazilian Archives of Biology and Technology. Curitiba, 39 (2): 393-400.

Costa, T.M. \& M.L. Negreiros-Fransozo. 1998. The reproductive cycle of Callinectes danae Smith 1869 (Decapoda, Portunidae) in Ubatuba region, Brazil. Crustaceana, Leiden, 71 (6): 615627.

Donaldson, W.E. \& A.E. AdAms. 1989. Ethogram of behavior with emphasis on mating for the tanner crab Chionoecetes bairdi Rathbun. Journal of Crustacean Biology, Lawrence, 9 (1): 37-53.

Fonteles-Filho, A.A. 1989. Recursos pesqueiros. Biologia e dinâmica populacional. Fortaleza, Imprensa Oficial do Ceará, $296 \mathrm{p}$.

González-Gurriarán, E.; L. Fernández; J. Freire \& R. Muiño. 1998. Mating and role of seminal receptacles in the reproductive biology of the spider crab Maja squinado (Decapoda, Majidae). Journal of Experimental Marine Biology and Ecology, Amsterdam, 220 (2): 269-285.

Hartnoll, R.G. 1982. Growth, p. 11-196. In: B.E. Bliss \& L.G. ABELE. (Eds). The biology of Crustacea: embriology, morphology and genetics. New York, Academic Press, 402p.

HARTNOLL, R.G. 1985. Growth, sexual maturity and reproductive output, p. 101-128. In: A. WENNER (Ed.). Crustacean Issues, 3: factors in adult growth. The Netherlands, Balkema Publishers, 375p.

Hartnoll, R.G. \& P. Gould. 1988. Brachyuran life history strategies and the optimization of egg production. Symposium of the Zoological Society of London,London, 59: 1-9.

Hattori, G.Y. \& M.A.A. Pinheiro. 2003. Fertilidade do caranguejo do mangue Ucides cordatus (Linnaeus), (Crustacea, Brachyura, Ocypodidae) em Iguape (São Paulo, Brasil). Revista Brasileira de Zoologia, Curitiba, 20 (2): 309-313.

HinEs, A.H. 1989. Geographic variation in size at maturity in 
brachyuran crabs. Bulletin of Marine Science, Miami, 45 (2): 356-368.

Iвама. 1995. Projeto co-gestão de manejo ambiental e desenvolvimento comunitário na APA de Guaraqueçaba, Paraná. Comunidades pesqueiras da APA de Guaraqueçaba: uma caracterização sóciocultural. Curitiba, IBAMA, SPVS, 53p.

Lestang, S.; N.G. Hall \& I.C. Potter. 2003. Influence of a deep artificial entrance channel on the biological characteristics of the blue swimmer crab Portunus pelagicus in a large microtidal estuary. Journal of Experimental Marine Biology and Ecology, Amsterdam, 295: 41-61

Mantelatto, F.L.M \& A. Fransozo. 1996. Size at sexual maturity in Callinectes ornatus (Brachyura, Portunidae) from the Ubatuba region (SP), Brazil. Nauplius, Rio Grande, 4: 29-38.

Mantelatto, F.L.M \& A. Fransozo. 1997. Fecundity of the crab Callinectes ornatus Ordway, 1863 (Decapoda, Brachyura, Portunidae) from the Ubatuba region, São Paulo, Brazil. Crustaceana, Leiden, 70 (2): 214-226.

Mantelatto, F.L.M \& J.M. Martinelli. 1999. Carapace widthweight relationships of Callinectes ornatus Ordway, 1863 (Brachyura, Portunidae) from the Ubatuba Bay, Brazil. Iheringia, Série Zoologia, Rio Grande, (87): 111-116.

MeLo, G.A.S. 1996. Manual de Identificação dos Brachyura (caranguejos e siris) do litoral brasileiro. São Paulo, Ed. Plêiade, FAPESP, 604p.

MeLo, G.A.S. 1999. Infraordem Brachyura. Siris e caranguejos: espécies marinhas e estuarinas, p. 415-485. In: L. BuckUP \& G. Bond-Buckup (Eds). Os crustáceos do Rio Grande do Sul. Porto Alegre, Editora UFRGS, 503p.

Negreiros-Fransozo. M.L \& A. Fransozo. 1995. On the distribuition of Callinectes ornatus Ordway, 1863 and Callinectes danae Smith, 1869 (Brachyura, Portunidae) in the Fortaleza Bay, Ubatuba, Brazil. Iheringia, Série Zoologia, Rio Grande, (79): 13-25.

Negreiros-Fransozo, M.L; F.L.M. Mantelatto \& A. Fransozo. 1999. Population biology of Callinectes ornatus Ordway, 1863 (Decapoda, Portunidae) from Ubatuba (SP), Brazil. Scientia Marina, Barcelona, 63 (2): 157-163.

PAUL, A.J. 1984. Mating frequency and variability of stored sperm in the tanner crab Chionoecetes bairdi (Decapoda, Majidae). Journal of Crustacean Biology, Lawrence, 4 (3): 375-381.

Pinheiro, M.A.A. \& A. Fransozo. 1998. Sexual maturity of the speckled swimming crab Arenaeus cribrarius (Lamarck, 1818) (Decapoda, Brachyura, Portunidae) in the Ubatuba littoral, São Paulo state, Brazil. Crustaceana, Leiden, 71 (4): 434452.
Pinheiro, M.A.A. \& A. Fransozo. 2002. Reproduction of the speckled swimming crab Arenaeus cribrarius (Brachyura: Portunidae) on the Brazilian coast near $23^{\circ} 30^{\prime}$ S. Journal of Crustacean Biology, Lawrence, 22 (2): 416-428.

Pinheiro, M.A.A. \& O.S.L. Terceiro. 2000. Fecundity and reproductive output of the speckled swimming crab Arenaeus cribrarius (Lamarck, 1818) (Brachyura, Portunidae). 2000. Crustaceana, Leiden, 73 (9): 1121-1137.

Pinheiro, M.A.A.; A. Fransozo. \& M.L. Negreiros-Fransozo. 1997. Dimensionamento e sobreposição de nichos dos Portunídeos (Decapoda, Brachyura), na Enseada da Fortaleza, Ubatuba, São Paulo, Brasil. Revista Brasileira de Zoologia, Curitiba, 14 (2): 371-378.

Pita, J.B.; E.S Rodrigues; R. Graça-Lopes; J.A.P. Coelho. 1985a. Observações bioecológicas sobre o siri Callinectes danae Smith 1869 (Crustacea, Portunidae), no Complexo Baía-Estuário de Santos, São Paulo, Brasil. Boletim do Instituto de Pesca, São Paulo, 12 (4): 35-43.

Pita, J.B.; E.S Rodrigues; R. Graça-Lopes; J.A.P. Coelho. 1985b. Levantamento preliminar da Família Portunidae (Crustacea, Decapoda, Brachyura) no Complexo Baía-estuário de Santos, São Paulo, Brasil. Boletim do Instituto de Pesca, São Paulo, 12 (3): 153-162.

Santos, S. \& M.L. Negreiros-Fransozo. 1997. Fecundity in Portunus spinimanus Latreille, 1819 (Crustacea, Brachyura, Portunidae) from Ubatuba, São Paulo, Brazil. Interciencia, Caracas, 22 (5): 259-263.

Santos, S. \& M.L. Negreiros-Fransozo. 1999. Reproductive cycle of the swimming crab Portunus spinimanus Latreille (Crustacea, Brachyura, Portunidae) from Ubatuba, São Paulo, Brazil. Revista Brasileira de Zoologia, Curitiba, 16 (4): 1183-1193.

SASTRY, A.N. 1983. Ecological aspects of reproduction, p. 179269. In: F.J. Verneerg; W.B. Vernberg. (Eds). The Biology of Crustacea. Environmental adaptations. Academic Press, New York., vol. 5, 471p.

Somerton, D.A. \& W.S. Meyers. 1983. Fecundity differences between primiparous and multiparous female Alaskan tanner crab (Chionoecetes bairdi). Journal of Crustacean Biology, Lawrence, 3 (2): 183-186.

Stone, R.P.; C.E. O'Clair \& T.C. Shirley. 1992. Seasonal migration and distribution of female red king crabs in a southeast Alaskan Estuary. Journal of Crustacean Biology, Lawrence, 12 (4): 546-560

Williams, A.B. 1974. The swimming crabs of the genus Callinectes (Decapoda: Portunidae). Fishery Bulletin, Seattle, 72 (3): 685-798.

Recebido em 05.VIII.2004; aceito em 25.V.2005. 